\title{
Integrated Soft Sensors and Elastomeric Actuators for Tactile Machines with Kinesthetic Sense
}

\author{
Sanlin S. Robinson ${ }^{\mathrm{a}}$, Kevin W. O’Brien ${ }^{\mathrm{b}}$, Huichan Zhao ${ }^{\mathrm{b}}$, Bryan N. Peele ${ }^{\mathrm{b}}$, Chris M. Larson ${ }^{\mathrm{b}}$, \\ Benjamin C. Mac Murray ${ }^{\mathrm{a}}$, Ilse M. van Meerbeek ${ }^{\mathrm{b}}$, Simon N. Dunham ${ }^{\mathrm{c}}$, Robert F. Shepherd ${ }^{\mathrm{b}, *}$
}

aDepartment of Materials Science and Engineering, Cornell University, 214 Bard Hall, Ithaca, NY 14853, USA

bSibley School of Mechanical and Aerospace Engineering, Cornell University, 105 Upson Hall, Ithaca, NY 14853, USA

'Department of Radiology, Weill Cornell Medical College, 413 E 69 ${ }^{\text {th }}$ St., Suite BRB-108, New York, NY 10021

* Correspondence to E-mail address: rfs247@cornell.edu

Keywords: stretchable sensors, soft robotics, PneuNets, hydrogels, and ionic liquids

\begin{abstract}
Human skin contains highly specialized deformation receptors that allow us to intuitively and effortlessly interpret our surroundings. These sensors help us to localize touch and determine the degree of contact pressure. In addition, the innate understanding of our own body posture is also due to these mechanoreceptors. This work demonstrates a synthetic sensory-motor analog that can be 3D printed, using direct ink writing (DIW) onto soft, fluidic elastomer actuators (FEAs). This 3D printing technique uses two inks — one that is an ionically conductive hydrogel and another that is an electrically insulating silicone - which is then patterned and photopolymerized into stretchable capacitive sensors. In this paper, these sensors are used to enable tactile sensing and kinesthetic feedback in a pneumatically actuated haptic device. This capacitive skin enabled the device to detect a compressive force from a finger press of $\sim 2 \mathrm{~N}$, and an internal pressurization of as low as $\sim 10 \mathrm{kPa}$.
\end{abstract}

\section{Introduction}

Human development, intelligence, and survival are intimately linked to tactile (i.e. touch and pressure) and kinesthetic (i.e. position and movement) feedback we receive from millions of 
mechanoreceptors embedded in our skin.[1,2] These highly specialized receptors are capable of transmitting signals through ionically conducting nerve fibers at rates of up to $70 \mathrm{~m} \mathrm{~s}^{-1}$.[2] When these sensors are deformed mechanically - perhaps by compression of the skin or contraction of a muscle - an action potential is transmitted along afferent pathways then processed in the central nervous system.[2] An appropriate response to the input stimulus is then returned along efferent neuronal pathways.[2] This sensory-motor coupling allows us to effortlessly perceive and interact with our environment, enabling the performance of complex tasks such as avoiding furniture in the dark or playing the piano. Due to skin's significant role in learning, tool manipulation, and gross motor coordination, the development of a synthetic analogue will enable new methods of feedback control in robotics, medical monitoring, and human-machine interfaces. Wearable[3] and implantable electronics[4] have been developed to integrate with human tissue, which is soft, three-dimensional, and mechanically dynamic.

Along with the development of stretchable electronics, the field of soft robotics has been gaining traction as a result of their ability to (i) interact gently with biological organisms,[5,6] (ii) easily conform to their surroundings,[7-9] and (iii) achieve complex motions using simple modes of actuation.[10-13] These robots are typically composed of hyperelastic materials (e.g. silicones) with large ultimate strains, $\Upsilon_{\text {ult }} \sim 400-700 \%$, that have embedded networks of pneumatic channels (PneuNets). These pneumatic actuators are powered through inflation, similar to a balloon. A critical limitation of soft robots is the availability of compliant and extensible sensors[14] that can (i) reliably detect external stimuli and internal actuation, (ii) be easily integrated with current manufacturing processes, (iii) function at the high strains encountered throughout actuation, and (iv) tolerate many actuation cycles without delamination.

To address these sensing deficiencies many groups have developed flexible and stretchable sensors that use changes in resistance,[15-17] capacitance,[18,19] light,[20] or resonant frequency[21,22] to detect deformation. Specifically, for soft robots that undergo large deformations, shape sensing is a critical need for feedback control (communicating with a central computer to ensure a machine task is being completed accurately). Therefore, we focus on sensing techniques using stretchable capacitors due to their high precision and sensitivity over large ranges of strain and pressure.[23,24]

Here we present a highly extensible sensing skin that we integrated with soft, pneumatic actuators via a 3D printing technique called direct ink writing (DIW; Figure 1). This skin enables soft machines to sense external stimuli as well as their own shape, thus creating a device that has 
both tactile and kinesthetic sense. Central to our approach is the development of two viscoelastic fluids - one that is ionically conductive (hydrogel elastomer precursor) and another that is electrically insulating (silicone elastomer precursor). We directly patterned these inks by extruding them in alternating layers through micronozzles of two different diameters, $d_{\text {hydr }}=330$ $\mu \mathrm{m}$ and $d_{s i}=250 \mu \mathrm{m}$, on our custom dual-head 3D printer. The two inks are yield-stress fluids that flow through our print heads at high shear rates and retain their shape after exiting the nozzles due to their viscoelastic properties. After extrusion, we used in-situ photopolymerization of the inks to chemically crosslink them into conductive hydrogels and insulating silicones with large ultimate strains, $\gamma_{\text {sil }}^{u l t}>350 \%$ and $\gamma_{\text {hydr }}^{u l t}>300 \%$ (Figures 2, S2). When patterned into alternating conductive and dielectric layers, these composite skins behave as stretchable capacitors, capable of transducing low mechanical stresses and large strains into electrical signals. We printed these stretchable capacitors onto soft, Pneumatically-powered Haptic Displays (PHD; Figures 1, S1) to provide sensory feedback control. We characterized the ability of the PHD to (i) detect touch (tactile sense), (ii) detect its own actuation (kinesthetic sense), and (iii) integrate these two senses for sensory-motor coupling.

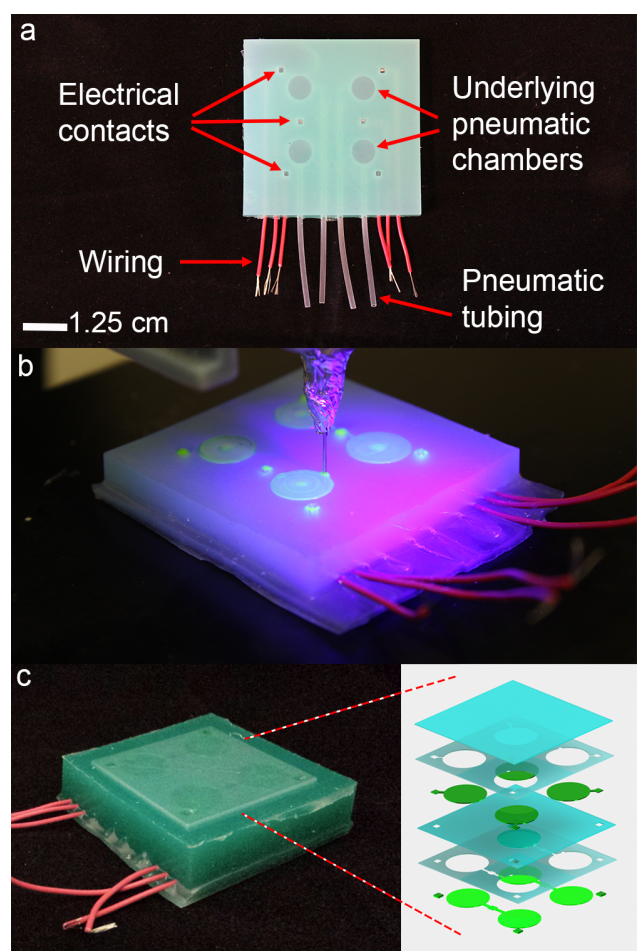

Figure 1. (a) Image of pneumatic haptic display with wells where electrical contacts will be formed, underlying pneumatic chambers, tubing and electrical wiring. (b) Image of printing stretchable sensors onto pneumatic haptic display. (c) Fully printed sensor on surface of pneumatic haptic display; (inset) Schematic of printed layers of conductive polyacrylamide (green) and insulating silicone (blue). 


\section{Experimental Design}

\subsection{Ink Preparation}

We chose to use DIW because it offers material flexibility, rapid design iteration, low cost,[25-27] and facile integration with previously established soft robot fabrication methods (i.e. replica molding[8] and rotational casting[9]). In addition, the rheological properties of many polymeric inks can be modified to enable this printing technique.[28-30] Specifically, both the shear thinning and yield stress properties of these complex fluids can be adjusted so the inks flow smoothly out of micronozzles and retain their shape after being printed. The shear thinning exponent $(n)$, storage $\left(\mathrm{G}^{\prime}\right)$ and loss $\left(\mathrm{G}^{\prime \prime}\right)$ moduli are primarily controlled by the high molecular weight polymer constituents.[30]

To tailor the rheology of the silicone elastomer precursor, we prepared blends of high and low molecular weight silicones (60 wt \% Nuvasil® Loctite 5039, 40 wt\% Wacker ${ }^{\circledR}$ Semicosil 912) together into a homogenous melt (details in supplemental information, SI). In this system, the high molecular weight polymer serves to adjust the yield stress, while the low molecular weight one polymerizes into a resilient matrix. We chose silicones because they have very high electrical resistivity (on the order of $10^{13} \Omega \cdot \mathrm{m}$ ), low leakage currents,[31] high strain to failure, and high resilience.[18,32]

We chose hydrogels because they can be made highly conductive, transparent, biocompatible, and stretchable.[18,33] We formulated the hydrogel elastomer precursor analogously to the silicone by combining high molecular weight polyacrylamide (PAAM 5-6 million $\mathrm{M}_{\mathrm{w}}$; 92560, Sigma Aldrich) and acrylamide monomer (AAM; Sigma Aldrich) in deionized water and ethylene glycol (EG; Sigma Aldrich). We also used N,N'methylenebisacrylamide (MBA; Sigma Aldrich) as the crosslinking agent and Darocur® 1173 (BASF) as the photoinitiator, ionic liquid (IL; 1-decyl-3-methylimidazolium chloride, Sigma Aldrich) served as the conductive medium. As the hydrogel is optically transparent, we used fluorescently labeled monomer (FOA; Fluorescein O-acrylate, Sigma Aldrich) for visualization of the ink during printing. We found that using $14 \mathrm{wt} \%$ AAM, 5.6 wt\% PAAM, $0.22 \mathrm{wt} \% \mathrm{MBA}$, $0.14 \mathrm{wt} \%$ Darocur ${ }^{\circledR} 1173,3.4 \mathrm{wt} \%$ IL, $0.1 \mathrm{wt} \%$ FOA, $56.2 \mathrm{wt} \%$ EG, and $20.3 \mathrm{wt} \%$ DI water resulted in the desired rheological and mechanical properties. 


\subsection{PHD Fabrication}

We fabricated silicone actuators using soft lithography as this process is simple and allows for rapid design iteration (details in SI). We then printed stretchable capacitors onto these pneumatic actuators as the skin of our PHD (Figures 1, S1). First we placed and leveled the prefabricated actuators on the bed of the 3D printer, then loaded the silicone and hydrogel inks into two separate syringes. By alternating layers of polyacrylamide and silicone inks we created planar arrays of capacitive sensors. The inks were exposed to high intensity UV radiation throughout the printing process. Once the capacitive skin was fully printed, no post exposure to UV light was necessary. We chose to overlay these sensors onto the actuators as an intuitive user interface to physically program the PHD; in addition, they enable us to detect the degree of actuation of individual chambers. Alternatively, if kinesthetic sensing is not needed, we can print the sensors anywhere, not necessarily on top of the actuators.

\section{Results and discussions}

\subsection{Material Characterization}

To be compatible with our pressure inputs $\left(\Delta P_{\text {sil }} \sim 270 \mathrm{kPa}\right.$ and $\left.\Delta P_{h y d r} \sim 70 \mathrm{kPa}\right)$ we tuned our materials to have viscosities less than $50 \mathrm{~Pa} \cdot \mathrm{s}$ at the shear rates for which we were printing (Figure 2a). Both inks displayed pronounced shear thinning above a critical shear rate of $\sim 0.1 \mathrm{~s}^{-1}$. This behavior indicates that above these shear rates, the entangled, long-chained polymers are aligning under the applied shear stress and will start to flow smoothly through micronozzles. Using Equation 1, we estimated the shear rate experienced by the ink at the nozzle walls during extrusion[34]:

$\dot{\gamma}_{w a l l}=\left(\frac{3+b}{4}\right) \frac{4 Q}{\pi R^{3}}$

where $b$ is the inverse of the shear thinning exponent, $Q=\boldsymbol{v} \boldsymbol{\pi} \boldsymbol{R}^{\mathbf{2}}$ is the volumetric flow rate, $\boldsymbol{v} \sim$ $4 \mathrm{~mm} \cdot \mathrm{s}^{-1}$ is the print speed, and $R$ is the nozzle radius. We determined the shear thinning exponent from our measured viscometry data to be $\boldsymbol{n}_{\boldsymbol{h y d r}}=0.287$ and $\boldsymbol{n}_{\text {sil }}=0.496$. We

calculated the shear rate experienced by these fluids at the nozzle to be $\dot{\gamma}_{w a l l}^{\text {hydr }} \sim 157 \mathrm{~s}^{-1}$ and $\dot{\boldsymbol{\gamma}}_{\boldsymbol{w a l l}}^{\text {sil }} \sim 160 \mathrm{~s}^{-1}$. At these shear rates, the ink viscosities are $\boldsymbol{\eta}_{\boldsymbol{h y d r}} \sim 7 \mathrm{~Pa} \cdot \mathrm{s}$ and $\boldsymbol{\eta}_{\boldsymbol{s i l}} \sim 17 \mathrm{~Pa} \cdot \mathrm{s}$. Ink 
elasticity is an important rheological parameter that can indicate whether ink will retain its printed shape before crosslinking. To determine the elasticity of these inks, we measured the shear elastic modulus $\left(\mathrm{G}^{\prime}\right)$ and viscous modulus $\left(\mathrm{G}^{\prime \prime}\right)$ at a frequency of $1 \mathrm{~Hz}$ (Figure $2 \mathrm{~b}$ ). The polyacrylamide ink has a plateau elastic modulus of $\boldsymbol{G}_{\boldsymbol{h y d r}}^{\mathbf{0}} \sim 350 \mathrm{~Pa}$ and a yield stress of $\boldsymbol{\sigma}_{\boldsymbol{y}}^{\boldsymbol{h y d r}} \sim$ $539 \mathrm{~Pa}$, while the silicone ink has $\boldsymbol{G}_{\boldsymbol{s i l}}^{\mathbf{0}} \sim 2250 \mathrm{~Pa}$ and a $\boldsymbol{\sigma}_{\boldsymbol{y}}^{\boldsymbol{s i l}} \sim 22 \mathrm{~Pa}$. We found that both inks display a solid-like response $\left(\mathrm{G}^{\prime}>\mathrm{G}^{\prime \prime}\right)$ at low shear stresses, which enables shape retention after extrusion and before being cured under UV illumination.

The skin of the PHD must withstand large strains, yet deform at low stresses. As the mechanical properties of the skin are dominated by the silicone, we measured its tensile properties (Figure 2c; details in SI). We also compared our printed material's mechanical properties to that of cast silicone (Figure 2c). At 100\% strain, the printed and cast silicones have elastic moduli of $E_{\text {print }} \sim 73 \mathrm{kPa}$ and $E_{\text {cast }} \sim 109 \mathrm{kPa}$, respectively. The lower elastic modulus of the printed silicone may indicate that the high molecular weight polymer remains in a prestretched state due to shear alignment from extrusion. 

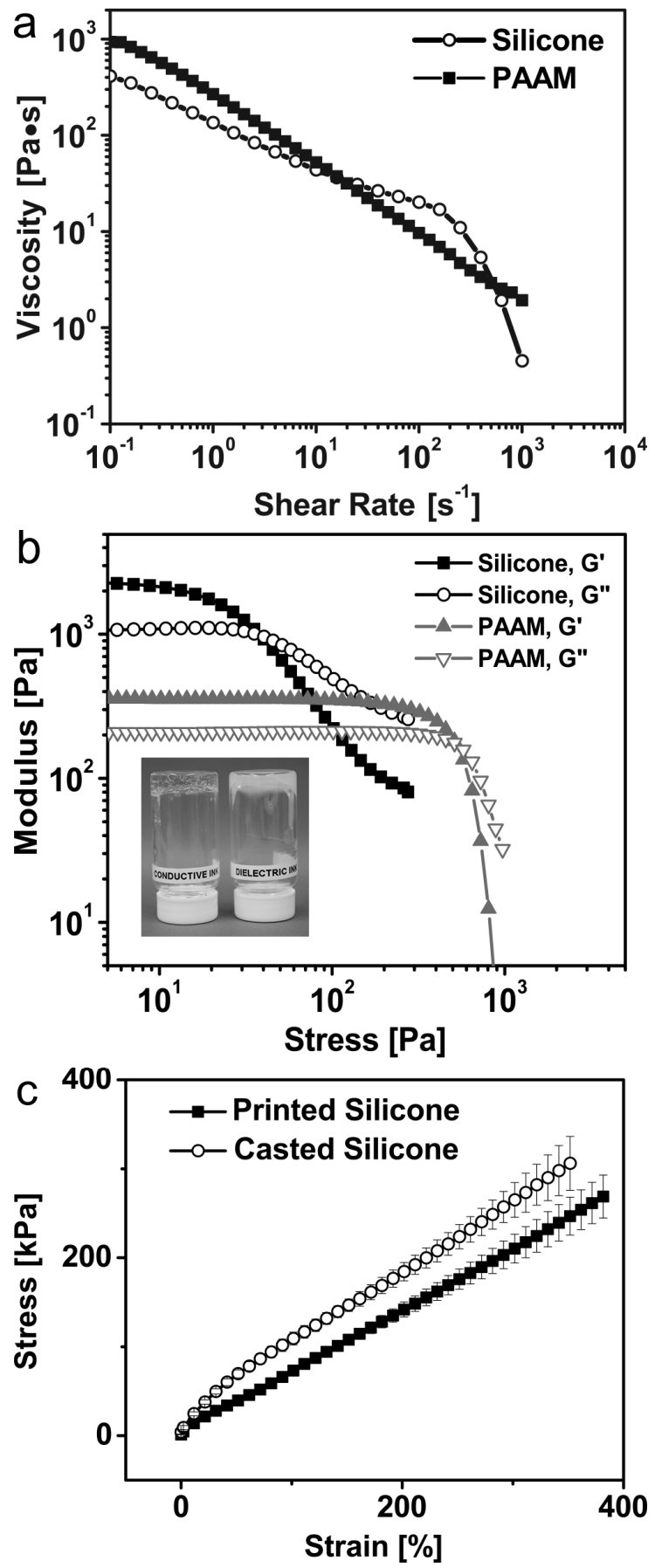

Figure 2. (a) Viscometry and (b) oscillatory rheology data for insulating silicone and conducting polyacrylamide (PAAM) inks; (inset) upturned vials of each ink demonstrate their elasticity. (c) Tensile data of 3D printed and cast silicone samples.

\subsection{Sensor Characterization}


Upon internal pressurization, the PHD's chambers expand in area causing the surface to dome; commensurately, the sensor area overlaying the chamber also increases while its thickness decreases, resulting in a change in capacitance, $\Delta \mathrm{C}$. We recorded the $\Delta \mathrm{C}$ using an LCR meter (Agilent 4284) while actuating a single chamber (Figure 3a, left) using a fixed volume syringe pump in series with a pressure gauge. Here we show that this behavior is consistent with that of a continuously deforming circular parallel plate capacitor (i.e., $C \sim A d^{-1}$, where A and $\mathrm{d}$ represent the electrode area and dielectric thickness, respectively). We first model the relationship between internal chamber pressure and the strain state of the sensor, and then reconcile that result with the expected change in capacitance as the sensor is stretched $C(\lambda)$.

To model $\mathrm{P}(\lambda)$, we employ three assumptions: (i) the membrane behaves linear elastically (Figure 2c) with an elastic modulus, E, (ii) the undeformed sensor is a circle and upon actuation deforms into a spherical cap with a radius of curvature $\mathrm{r}$, and deflection $\mathrm{h}$, and (iii) the elastomeric membrane is incompressible giving rise to principle stretches of $\lambda_{1}=\lambda_{2}=\lambda, \lambda_{3}=$ $\lambda^{-2}$, and principal stresses of $\sigma_{1}=\sigma_{2}=\sigma, \sigma_{3}=0$. We first balance the forces from the internal pressure $(P)$ acting on the membrane with the induced reaction forces within it.

$P \cdot\left(\pi r_{0}^{2}\right)=T \cdot \sin \theta$

$T=\sigma \cdot\left(2 \pi r_{0}\right) \cdot t$

$\sin \theta=\frac{r_{0}}{r}$

Where $r_{0}$ is the radius of the undeformed sensor, $T$ is the stretching force, $t$ is the nominal thickness of the membrane (see Figure S6). The pressure can therefore be expressed as follows:

$P=\frac{2 \sigma \cdot t}{r}$

Given assumptions $2 \& 3$ we can relate $\lambda$ to $r$, $t$, and $t_{0}$ as follows: 
$\frac{A}{A_{0}}=\frac{2 \pi r h}{\pi r_{0}^{2}}=\lambda^{2} \quad$ and $\quad \frac{t}{t_{0}}=\lambda^{-2}$

We now invoke a geometrical argument that relates $\mathrm{r}$ to $\mathrm{r}_{0}$ and $\mathrm{h}$, both of which are measurable quantities.

$r^{2}=r_{0}^{2}+(r-h)^{2}$

Combining Equations (2)-(5) with Hooke's law, $\sigma=E(\lambda-1)$, yields the following relation:

$P(\lambda)=\frac{4 t_{0} E(\lambda-1)\left(\lambda^{2}-1\right)^{1 / 2}}{r_{0} \lambda^{4}}$

The circular capacitive sensor is designed such that the in the fully actuated state (i.e., a hemisphere), it covers the underlying chamber completely. The nominal radius of the sensor, $\mathrm{r}_{\mathrm{C} 0}$, is thus larger than $\mathrm{r}_{0}$. To model the change in capacitance as accurately as possible, therefore, we bifurcate the capacitance equation into two parts - the first models the outer region that is bounded by $\mathrm{r}_{\mathrm{C} 0}$ and $\mathrm{r}_{0}\left(\mathrm{C}_{0}\right)$, and the center portion bounded by $\mathrm{r}_{0}\left(\mathrm{C}_{1}\right)$. Our equation for capacitance is:

$C=C_{0}+C_{1}$

where $C_{0}=\varepsilon_{r} \varepsilon_{0} \frac{\pi\left(r_{C 0}^{2}-r_{0}^{2}\right)}{t_{C 0}}$

and $C_{1}=\varepsilon_{r} \varepsilon_{0} \frac{2 \pi r h}{t_{C}}=\varepsilon_{r} \varepsilon_{0} \pi \frac{r_{0}{ }^{2}}{t_{C 0}} \lambda^{4}$

Combining relations in Equation 7 yields the relationship between $\lambda$ and $\mathrm{C}$ :

$\lambda=\left(\frac{C-C_{0}}{\varepsilon_{r} \varepsilon_{0} \pi \frac{r_{0}^{2}}{t_{C 0}}}\right)^{1 / 4}$ 
Finally, we substitute $\lambda(C)$ in Equation 10 into Equation 6 to derive $C(P)$. We solve for $C(P)$ numerically in MATLAB to obtain the plot shown in Figure 3a, left. Table 1 lists the parameters used in the model. We observe that the predicted relationship between $\mathrm{C}$ and $P$ is in general agreement with the measured values, confirming that our deformable capacitor follow $\mathrm{C} \sim \mathrm{A} / \mathrm{d}$ behavior. The slight deviation between the predicted and measured values at pressures $>25 \mathrm{kPa}$ indicates that our bifurcation scheme could be further refined.

Table 1. Model Parameters

\begin{tabular}{|c|c|c|c|c|c|c|c|c|}
\hline$P$ & $\lambda$ & $\sigma$ & $r$ & $t$ & $h$ & $r_{0}$ & $t_{0}$ & $E$ \\
\hline $\begin{array}{c}\text { Internal } \\
\text { pressure }\end{array}$ & Stretch & Stress & Radius & Thickness & Height & $\begin{array}{c}\text { Chamber } \\
\text { radius }\end{array}$ & $\begin{array}{c}\text { Initial } \\
\text { thickness }\end{array}$ & $\begin{array}{c}\text { Young's } \\
\text { modulus }\end{array}$ \\
\hline
\end{tabular}

\begin{tabular}{|c|c|c|c|c|c|}
\hline$C$ & $r_{C 0}$ & $t_{C}$ & $t_{C 0}$ & $\varepsilon_{r}$ & $\varepsilon_{0}$ \\
\hline \multirow{3}{*}{ Capacitance } & Initial & Sensor & Initial & Relative & Dielectric \\
& Sensor & sensor & $\begin{array}{c}\text { permittivity } \\
\text { thickness }\end{array}$ & constant \\
& radius & & thickness & & \\
\hline
\end{tabular}

\begin{tabular}{|l|l|l|l|l|l|l|}
\hline$r_{0}$ & $t_{0}$ & $E$ & $r_{C 0}$ & $t_{C 0}$ & $\varepsilon_{r}$ & $\varepsilon_{0}$ \\
\hline $4.0 \mathrm{~mm}$ & $3.7 \mathrm{~mm}$ & $68.9 \mathrm{kPa}$ & $6.0 \mathrm{~mm}$ & $0.33 \mathrm{~mm}$ & 2.2 & $8.854 \times 10^{-12} \mathrm{~F} \cdot \mathrm{m}^{-1}$ \\
\hline
\end{tabular}

Next, we demonstrated the ability of the PHD to transduce external touch into an electrical signal by repeatedly pressing on a single sensor cell 99 times (Figures 3a, right, S3). The average force delivered during the press of a sensor cell is approximately $2 \mathrm{~N}$ (SI text), this force causes a detectable $\Delta \mathbf{C} \sim 1 \mathrm{pF}$. As our goal was not to precisely measure the force a finger applies but to demonstrate the sensitivity to touch of the PHD, we used our own fingers instead of a machine; consequently there is variation in the measured capacitance between the peaks.

Additionally, using a pressurized air dispensing system, we actuated a single chamber of our pneumatic display while measuring the change in capacitance (Figures 3b, 3c). We applied incremental pressures of air to the chamber, we then released the pressure and allowed it to deflate and repeated this cycle (Figure $3 \mathrm{c}$ ). We only tested up to $38 \mathrm{kPa}$, above that pressure we start to observe deformation of the hydrogel electrode layers. No discernable capacitance change was observed until at least $\Delta \mathrm{P} \sim 10 \mathrm{kPa}$ was delivered to the chamber (Figures $3 \mathrm{~b}, 3 \mathrm{c}$ inset). Since 
we were inflating and deflating manually, the time for each actuation varied, thus the peak widths vary. The shapes of the peaks continue to evolve after initial pressurization due to the viscoelastic nature of the materials.
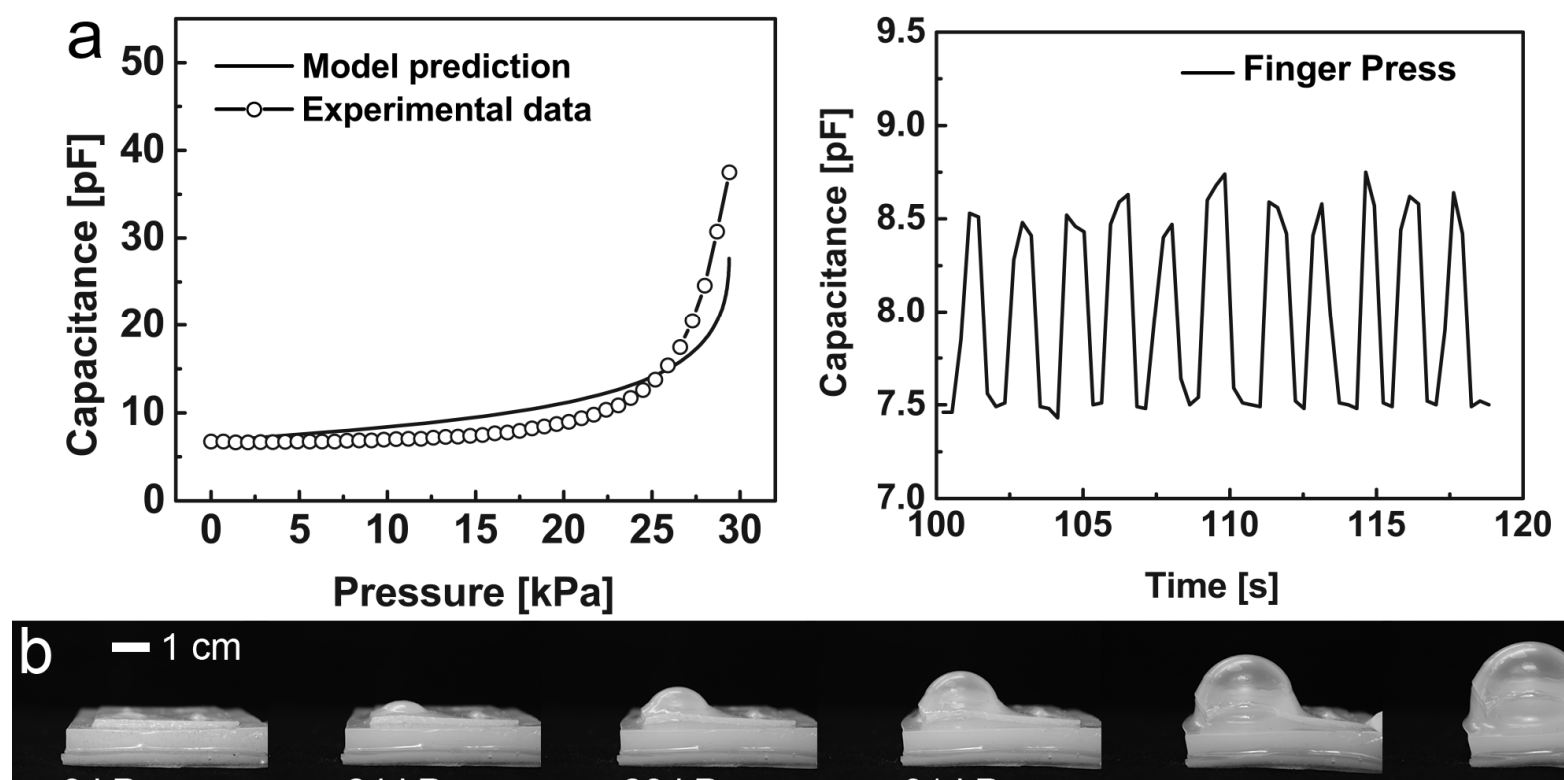

$0 \mathrm{kPa}$

$24 \mathrm{kPa}$

$28 \mathrm{kPa}$

$31 \mathrm{kPa}$

$35 \mathrm{kPa}$

$38 \mathrm{kPa}$

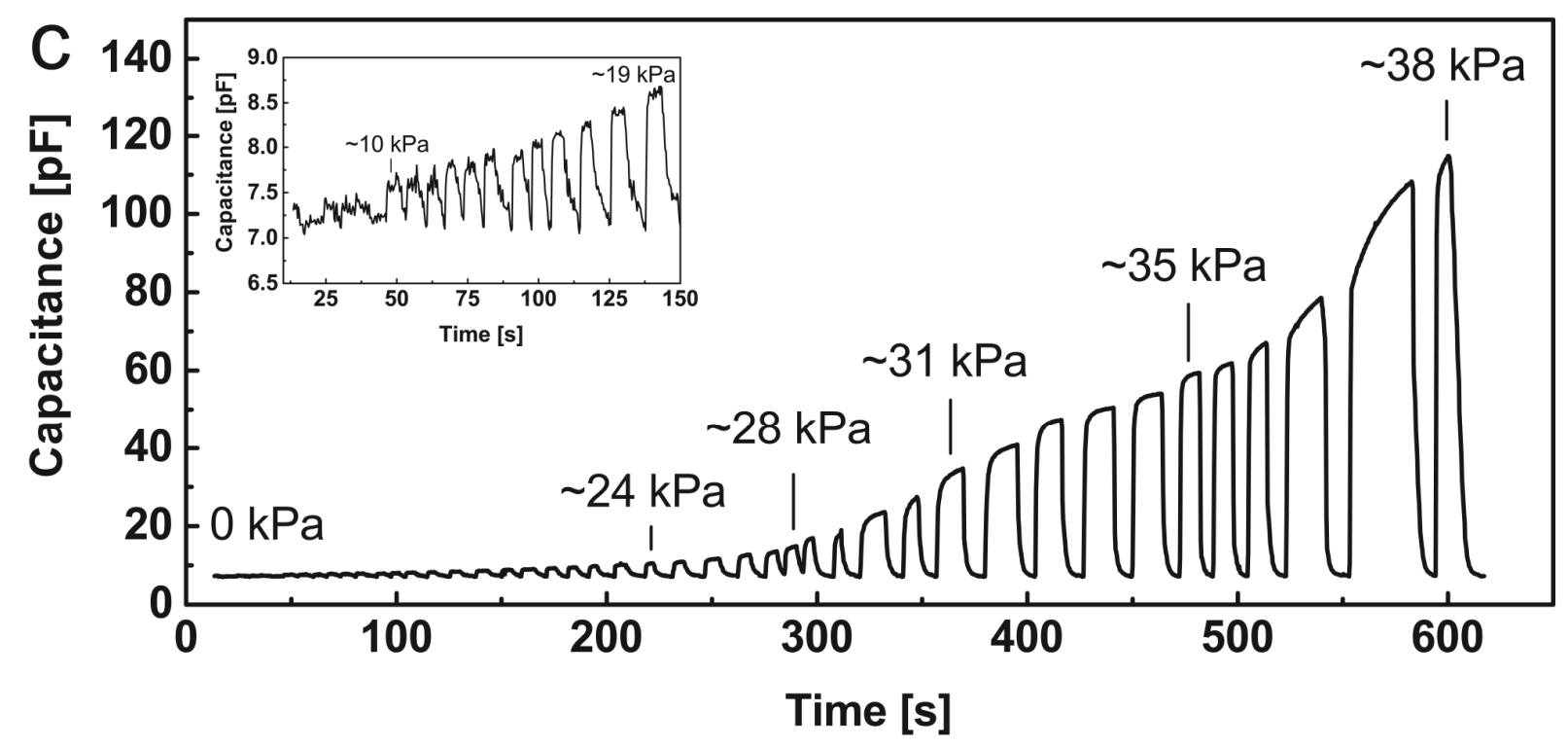

Figure 3. (a) Left, the measured (circles) and theoretical (line) capacitance as a function of inflation pressure. Right, repeatability of capacitor to sense stimulus when pressed by a finger. (b) Left to right, images of actuation at different applied pressures. (c) Capacitance dependence on inflation and deflation of actuator at increasing pressures; (inset) lower capacitance range magnified.

\subsection{PHD Device Demonstration}


To demonstrate our printed sensor array's ability to encode and read haptic information, we programmed a five-digit sequence into the PHD (SI text). We pressed on the individual capacitive buttons in an arbitrary sequence, sending a signal to an off-board microcontroller's memory (Arduino Mega 2560). The microcontroller then played back the recorded sequence through pneumatic actuation of the individual chambers (Figure 4; supplemental video, SV1). We numbered the sensors one through four, and then punched in the code 2-4-3-2-1 (Figure 4a). The PHD then individually inflated each underlying chamber associated with the sensor input (Figure 4b). The PHD detected the $\Delta \mathbf{C}$ of both the input stimulus (tactile sense) and output actuation (kinesthetic sense; Figure 4c). We note that the peaks from the input are sharp and output are broad due to the duration of the different events.
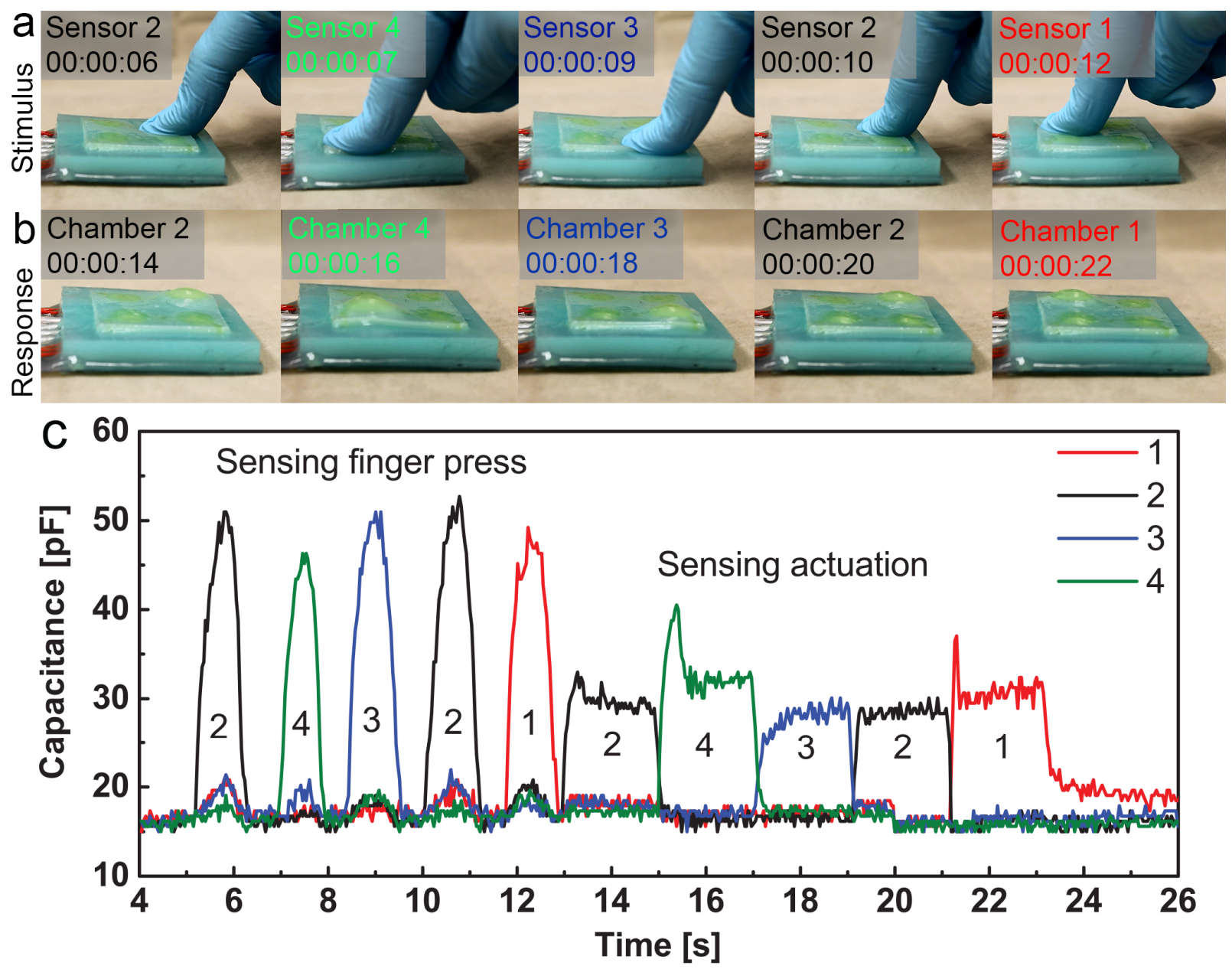

Figure 4. (a) Left to right, digital images of inputting a code (2-4-3-2-1) using finger to push on the sensor. (b) Left to right, pneumatic haptic display playing back the same code (2-4-3-2-1). (c) Capacitance change of sensor as the code is being input, and then played back through pneumatic actuation. Please see SV1 for video of programming and display sequence. 


\section{Conclusion}

In summary, we have developed an inexpensive, hyperelastic, and printable skin for the sensory-motor coupling of soft machines. By tuning the rheological properties of elastomer precursors we formulated viscoelastic inks, which we patterned directly onto the actuators of our PHD. This capacitive skin enabled the PHD to detect a compressive force of $\sim 2 \mathrm{~N}$, and an internal pressurization of as low as $\sim 10 \mathrm{kPa}$. As far as we know this is the first demonstration of a completely soft, feedback controlled haptic interface. As the majority of the mating surface between the skin and actuators is silicone, the device exhibited excellent adhesion and no delamination occurred even when high pressures (up to $\sim 65.5 \mathrm{kPa}$ ) were applied to the actuating chambers. At these pressures, however, the inextensible sealing layer delaminated from the bottom of the PHD; this issue is general to PneuNets operated at high pressure.

While the hydrogels used here have some amount of solvent and are thus somewhat sensitive to environmental conditions, solvent-free systems, such as the polyacrylic acid ones recently demonstrated,[35] could be used to mitigate this issue. While this is a 2D array of capacitors, 3D systems are possible with more complicated CAD design. It is also possible to miniaturize these capacitors by increasing the dielectric constant of the insulating material through the addition of fillers and the use of smaller nozzle diameters for decreased layer thickness. With these two steps we can more accurately replicate the natural ability of biological skin to localize touch sensations. The reliable, low cost, and flexible design space using DIW of tactile and kinesthetic sensors will improve robotic technologies - which are normally confined to perform repetitive tasks in structured environments (i.e. mobile industry) — by allowing humans to work more closely with machines in applications such as search and rescue and domestic care. This same sensor technology could also improve the quality of life for amputees by enabling intuitive use and automatic adjustment of prosthetic devices.

\section{Acknowledgements}

This work was supported in part by the Air Force Office of Scientific Research under award number FA9550-15-1-0160, by a 3M Non-Tenured Faculty Award, and by the National Science Foundation Graduate Research Fellowship under Grant No. DGE-1144153. This work made use 
of the Cornell Center for Materials Research Facilities supported by the National Science Foundation under Award Number DMR-1120296.

\section{References}

[1] R. Pfeifer, M. Lungarella, F. Iida, Commun. ACM 2012, 55, 76.

[2] A. C. Guyton, Textbook of Medical Physiology, W.B. Saunders Company, 1981.

[3] G. Frediani, D. Mazzei, D. E. De Rossi, F. Carpi, Front Bioeng Biotechnol 2014, 2, 31.

[4] D.-H. Kim, R. Ghaffari, N. Lu, J. A. Rogers, Annu. Rev. Biomed. Eng. 2012, 14, $113-$ 128.

[5] P. Polygerinos, S. Lyne, Zheng Wang, L. F. Nicolini, B. Mosadegh, G. M. Whitesides, C. J. Walsh, in 2013 IEEE/RSJ International Conference on Intelligent Robots and Systems (IROS 2013), Ieee, 2013, pp. 1512-1517.

[6] R. V. Martinez, J. L. Branch, C. R. Fish, L. Jin, R. F. Shepherd, R. M. D. Nunes, Z. Suo, G. M. Whitesides, Advanced Materials 2012, 25, 205-212.

[7] F. Ilievski, A. D. Mazzeo, R. F. Shepherd, X. Chen, G. M. Whitesides, Angew. Chem. Int. Ed. 2011, 50, 1890-1895.

[8] R. F. Shepherd, F. Ilievski, W. Choi, S. A. Morin, A. A. Stokes, A. D. Mazzeo, X. Chen, M. Wang, G. M. Whitesides, Proceedings of the National Academy of Sciences 2011, 108, 20400-20403.

[9] H. Zhao, Y. Li, A. Elsamadisi, R. Shepherd, Extreme Mechanics Letters 2015, 3, 89-104.

[10] C. M. Schumacher, M. Loepfe, R. Fuhrer, R. N. Grass, W. J. Stark, RSC Adv. 2014, 4, 16039.

[11] M. T. Tolley, R. F. Shepherd, B. Mosadegh, K. C. Galloway, M. Wehner, M. Karpelson, R. J. Wood, G. M. Whitesides, Soft Robotics 2014, 1, 213-223.

[12] A. D. Marchese, C. D. Onal, D. Rus, Soft Robotics 2014, 1, 75-87.

[13] B. Mosadegh, P. Polygerinos, C. Keplinger, W. Sophia, R. F. Shepherd, U. Gupta, J. Shim, K. Bertoldi, C. J. Walsh, G. M. Whitesides, Advanced Functional Materials 2014, 24, 2163-2170.

[14] D. Rus, M. T. Tolley, Nature 2015, 521, 467-475.

[15] Yong-Lae Park, Bor-Rong Chen, R. J. Wood, IEEE Sensors J. 2012, 12, 2711-2718.

[16] J. T. Muth, D. M. Vogt, R. L. Truby, Y. Mengüç, D. B. Kolesky, R. J. Wood, J. A. 
Lewis, Advanced Materials 2014, 26, 6307-6312.

[17] C. Yan, J. Wang, W. Kang, M. Cui, X. Wang, C. Y. Foo, K. J. Chee, P. S. Lee, Advanced Materials 2013, 26, 2022-2027.

[18] J.-Y. Sun, C. Keplinger, G. M. Whitesides, Z. Suo, Advanced Materials 2014, 26, 76087614.

[19] A. Frutiger, J. T. Muth, D. M. Vogt, Y. Mengüç, A. Campo, A. D. Valentine, C. J. Walsh, J. A. Lewis, Advanced Materials 2015, 27, 2440-2446.

[20] M. Ramuz, B. C. K. Tee, J. B. H. Tok, Z. Bao, Advanced Materials 2012, 24, 32233227.

[21] M. Kubo, X. Li, C. Kim, M. Hashimoto, B. J. Wiley, D. Ham, G. M. Whitesides, Advanced Materials 2010, 22, 2749-2752.

[22] M. Rashed Khan, G. J. Hayes, J.-H. So, G. Lazzi, M. D. Dickey, Appl. Phys. Lett. 2011, 99, 013501.

[23] M. Kollosche, H. Stoyanov, S. Laflamme, G. Kofod, Journal of Materials Chemistry 2011, 21, 8292-8294.

[24] W. Y. Du, S. W. Yelich, Sensors and Transducers 2008, 1-25.

[25] J. E. Smay, G. M. Gratson, R. F. Shepherd, Advanced Materials 2002, 14, 1279-1283.

[26] D. Therriault, R. F. Shepherd, S. R. White, J. A. Lewis, Advanced Materials 2005, 17, $395-399$.

[27] J. C. Conrad, S. R. Ferreira, J. Yoshikawa, R. F. Shepherd, B. Y. Ahn, J. A. Lewis, Current Opinion in Colloid \& Interface Science 2011, 16, 71-79.

[28] R. A. Barry III, R. F. Shepherd, J. N. Hanson, R. G. Nuzzo, P. Wiltzius, J. A. Lewis, Advanced Materials 2009, 21, 2407-2410.

[29] J. N. Hanson Shepherd, S. T. Parker, R. F. Shepherd, M. U. Gillette, J. A. Lewis, R. G. Nuzzo, Advanced Functional Materials 2010, 21, 47-54.

[30] J. A. Lewis, Advanced Functional Materials 2006, 16, 2193-2204.

[31] J. Biggs, K. Danielmeier, J. Hitzbleck, J. Krause, T. Kridl, S. Nowak, E. Orselli, X. Quan, D. Schapeler, W. Sutherland, J. Wagner, Angew. Chem. Int. Ed. 2013, 52, 94099421.

[32] P. Brochu, Q. Pei, Macromol. Rapid Commun. 2010, 31, 10-36.

[33] C. Keplinger, J.-Y. Sun, C. C. Foo, P. Rothemund, G. M. Whitesides, Z. Suo, Science 2013, 341, 984-987. 
[34] J. Bruneaux, D. Therriault, M.-C. Heuzey, J. Micromech. Microeng. 2008, 18, 115020.

[35] B. Chen, J. J. Lu, C. H. Yang, J. H. Yang, J. Zhou, Y. M. Chen, Z. Suo, ACS Appl. Mater. Interfaces 2014, 6, 7840-7845. 